\section{ANTICOAGULATION THERAPY SAFETY OF REG1 ON THE RADAR}

New data from the RADAR phase Ilb trial suggest that the REG1 anticoagulation system-consisting of the single-stranded RNA factor IXa inhibitor, pegnivacogin, and the reversal agent, anivamersenis safe for use in patients with acute coronary syndromes (ACS) undergoing percutaneous coronary interventions. REG1 administration facilitated the prompt removal of femoral sheaths following cardiac catheterization.

Patients with non-ST-segment elevation ACS were randomly assigned to REG1 $(n=479)$ or heparin $(n=161)$ before planned cardiac catheterization via femoral access. Patients in the REG1 group underwent sheath removal $10 \mathrm{~min}$ after the procedure and received varying postprocedural doses of anivamersen to achieve $25-100 \%$ reversal of anticoagulation, whereas those in the heparin group underwent sheath removal as per the standard of care.

At 30-day follow-up, a substantial increase in total and major bleeds occurred in patients who received REG1 with 25\% anticoagulation reversal compared with those who received heparin. However, the incidence of bleeds in patients who received $\geq 50 \%$ reversal was similar to that of patients in the heparin group. High levels of anticoagulation reversal did not increase the risk of acute $(<24 \mathrm{~h})$ or short-term (30 days) thrombotic events.

Enrollment in the trial was stopped after three patients experienced allergiclike reactions soon after pegnivacogin administration. These reactions might be related to prior exposure of the affected individuals to polyethyleneglycol, which was attached to the nucleic-acid component of pegnivacogin to improve drug stability.

The trial investigators write "near complete inhibition of factor IXa with pegnivacogin, followed by anivamersenmediated reversal, may provide a strategy that permits effective periprocedural anticoagulation without increased bleeding despite immediate postprocedure sheath removal". However, they emphasize that further trials are required to evaluate the safety and efficacy of the REG1 system in comparison with other high-intensity, short-term anticoagulation strategies.

Ellen F. Carney

Original article Povsic, T. J. et al. A phase 2, randomized, partially blinded, active-controlled study assessing the efficacy and safety of variable anticoagulation reversal using the REG1 system in patients with acute coronary syndromes: results of the RADAR trial. Eur. Heart J. doi:10.1093/eurheartj/ehs232 